\title{
Représentants, mandataires et commettants : Robespierre, la relation fiduciaire et le droit à l'existence matériel et politique
}

\author{
Representantes, agentes y principales: Robespierre, la relación \\ fiduciaria y el derecho a la existencia material y política
}

\author{
Representatives, agents and principals: Robespierre, the fidu- \\ ciary relationship and the right to material and political existence
}

YANNICK BOSC

\begin{abstract}
Résumé : Pendant la Révolution française, le travail politique du « côté gauche », dont Robespierre est l'un des porte-parole, consiste à mettre en œuvre les principes de la Déclaration des droits de l'homme et du citoyen. Ceux-ci impliquent que le peuple souverain puisse contrôler l'exercice du pouvoir de ses mandataires. Ils ont pour fonction de garantir le droit naturel de l'homme à l'existence qui est la principale condition de la liberté et la raison d'être de la république.

Mots clés : Citoyenneté - Droit à l'existence Droits naturels - Propriété - Républicanisme Souveraineté populaire.
\end{abstract}

\begin{abstract}
During the French Revolution, the political work of the «left side», of which Robespierre was one of the spokespersons, consisted in implementing the principles of the Declaration of the Rights of Man and of the Citizen. These imply that the sovereign people can control the exercise of the power of their agents. Their function is to
\end{abstract}

guarantee the natural right of man to existence which is the main condition of freedom and the raison d'être of the republic.

Keywords : Citizenship - Natural rights - Popular sovereignty - Property - Republicanism Right to existence.

Resumen: Durante la Revolución Francesa, el trabajo político del "lado izquierdo", del cual Robespierre fue uno de los portavoces, consistió en implementar los principios de la Declaración de los Derechos del Hombre y del Ciudadano. Esto implica que el pueblo soberano puede controlar el ejercicio del poder de sus agentes. Su función es garantizar el derecho natural del hombre a la existencia, que es la condición principal de la libertad y la razón de ser de la república.

Palabras clave: Ciudadanía - Derecho a la existencia - Derechos naturales - Propiedad - Republicanismo - Soberanía popular.

Recibido: 08/06/2020. Aceptado: 17/06/2020. Cómo citar este artículo: Bosc, Y. (2020). Représentants, mandataires et commettants : Robespierre, la relation fiduciaire et le droit à l'existence matériel et politique. Daimon . Revista Internacional de Filosofía, (81), 67-81. https://doi.org/10.6018/daimon.431371

* Université de Rouen Normandie (GRHis). Profesor de historia moderna. Contacto: yannick.bosc@univ-rouen. fr. Líneas de investigación: Revolución Francesa; republicanismos y derecho natural; economía política republicana; propiedad y bienes comunes; historiografía de la revolución francesa. Publicaciones recientes: Yannick Bosc, Le peuple souverain et la démocratie. Politique de Robespierre, Paris, Éditions Critiques, 2019; Yannick Bosc et Emmanuel Faye (dir.), Hannah Arendt, la révolution et les droits de l'homme, Paris, Kimé, 2019. 
Dans la tradition républicaine, le citoyen est à la fois celui qui gouverne et qui est gouverné, une condition sans laquelle il ne serait pas libre, mais soumis à la domination de ceux qui établissent la loi. Aussi, la question du pouvoir délégué comme celle des modalités de cette délégation y est centrale. Le républicanisme de Robespierre s'inscrit plus spécifiquement dans la tradition démocratique du républicanisme - distincte de l'aristocratique - qui à l'époque moderne est étroitement liée à celle du droit naturel. Pendant la Révolution française, celle-ci s'exprime dans les Déclarations des droits de l'homme et du citoyen de 1789 et 1793 . Cette tradition, d'abord affirmée dans l'Angleterre révolutionnaire du XVII ${ }^{\mathrm{e}}$ siècle, se reconnaît en particulier dans le fait que le peuple souverain est considéré comme le seul apte à juger l'exerce du pouvoir de ses mandataires ou commis, que ceux-ci soient « le prince » ou « la puissance législative » : «Qui, écrit Locke, jugera si le Prince, ou la puissance législative, passe l'étendue de son pouvoir et de son autorité ? [...] je réponds, que c'est le peuple qui doit juger de cela. En effet, qui est-ce qui pourra mieux juger si l'on s'acquitte bien d'une commission, que celui qui l'a donnée, et qui par la même autorité, par laquelle il a donné cette commission, peut désapprouver ce qu'aura fait la personne qui l'a reçue, et ne se plus servir d'elle, lorsqu'elle ne se conforme pas à ce qui lui a été prescrit (Locke, 1690, § 240) ? »Cette relation que décrit Locke entre le peuple et ses commis est celle de la fidéicommission. Le peuple - c'est-à-dire l'association de citoyens libres et égaux en droits - étant le « principal » ou fidéicommettant ou commettant, celui qui est chargé de la commission étant « l'agent », le fidéicommissaire ou mandataire » (trustee) (Domènech, 2009, 19).

La fidéicommission repose sur un rapport asymétrique en faveur du commettant, celui-ci pouvant se séparer unilatéralement de son commis quand il le désire. Il ne s'agit pas d'un contrat (Domènech, Ibid.). En revanche, la Déclaration des droits de l'homme et du citoyen est un contrat passé entre citoyens, fondé comme tout contrat sur un rapport symétrique entre les contractants (aucune partie ne peut se dégager unilatéralement des termes du contrat). Elle n'est pas un contrat entre les « gouvernants » et les « gouvernés », comme on le croit souvent, puisque leurs rapports relèvent de la fidéicommission.

Les mandataires du peuple ont pour fonction de mettre en œuvre le contrat (la Déclaration) passé entre les citoyens et ceux-ci doivent le vérifier : «Chez un peuple libre et éclairé, écrit Robespierre, le droit de censurer les actes législatifs est aussi sacré que la nécessité de les observer est impérieuse » $(\mathrm{IV}, 146)^{1}$. Un grand nombre de ses interventions consiste à le rappeler. Nous verrons que pour lui, la confiance du peuple souverain vis à vis de ceux qui le représentent dépend de leur capacité à s'acquitter de cette commission, en d'autres termes de leur capacité à mettre en œuvre les principes de la Déclaration qui constituent la république, la chose commune. A ses yeux - et au-delà pour le mouvement populaire et ses porte-parole -, les plus essentiels de ces principes résident dans la garantie du droit naturel à l'existence, que celui-ci soit matériel (l'accès aux biens qui permettent de vivre) ou politique (l'exercice de la citoyenneté), les deux étant intimement liés, comme nous le constaterons : «L'homme, dit Robespierre, est citoyen par la nature ; personne ne saurait lui arracher ce droit, qui est inséparable de celui qu'il a d'exister sur la terre » (VI, 553)². Le Gouvernement

1 Le Défenseur de la Constitution, n5, Juin 1792.

2 « Sur les droits politiques des indigents », 23 octobre 1790. 
révolutionnaire, qui consiste notamment dans le contrôle populaire de la propriété des biens qui permettent d'exister, est un exemple de mise en œuvre de ce droit à l'existence matériel et politique où la relation fiduciaire joue un rôle cardinal.

Robespierre nous permet ainsi d'aborder la question centrale que l'on tente de résoudre pratiquement - ou d'esquiver selon les camps - pendant la Révolution française : comment représenter politiquement un peuple déclaré souverain ? Avant de traiter des liens qui chez Robespierre unissent le droit à l'existence et la représentation politique, nous nous intéresserons d'une part, à la manière dont il conçoit la délégation de pouvoir par le peuple souverain et, d'autre part, au sens qu'il donne aux fonctions de représentant, de mandataire, de délégué ou encore de commis du peuple, qui ont en commun d'être les dépositaires de la confiance du peuple et des termes dont le contrôle est au centre des luttes politiques (Guilhaumou, 1989) en particulier dans les premières années de la Révolution au cours desquelles le statut du roi est en jeu.

\section{Les dépositaires de la confiance du peuple}

Le 10 août 1791, au nom du comité de Constitution, Thouret présente à la Constituante le titre III du projet de Constitution, consacré aux pouvoirs publics. Les deux premiers articles sont conçus en ces termes :

«Art. 1. La souveraineté est une, indivisible et appartient à la Nation ; aucune section du peuple ne peut s'en attribuer l'exercice».

«Art 2. La Nation, de qui seule émanent tous les pouvoirs, ne peut les exercer que par délégation. La Constitution française est représentative ; les représentants sont le corps législatif et le roi ».

La presse rapporte que Robespierre demande la parole car il y a, dit-il, « dans le titre soumis à votre délibération beaucoup d'expressions équivoques et de mots qui altèrent le véritable sens et l'esprit de votre constitution ». Il se propose donc de « rectifier ces mots » afin de « rendre d'une manière claire les principes de votre constitution » (VII, 612), c'est-à-dire les principes de la Déclaration des droits de l'homme et du citoyen adoptée le 26 août 1789.

Il s'agit en particulier de la formulation de l'article 2 selon lequel la Nation ne peut exercer ses pouvoirs que par délégation : «Je soutiens, dit Robespierre, que les différents pouvoirs de la Nation ne sont autre chose que les parties constitutives de la souveraineté ; et comme la souveraineté est inaltérable, ces pouvoirs sont aussi inaliénables (VII, 614-615). »

Contrairement à ce qu'insinue Thouret qui l'interpelle, Robespierre ne considère pas que la nation ne peut pas déléguer ses pouvoirs : «Je n'ai point dit cela, lui répond-il. J'ai dit simplement que la Nation ne pouvait pas déléguer ses pouvoirs à perpétuité dans le sens du comité, ce qui est une aliénation» (VII, 614).

Robespierre suspecte à juste titre la majorité de la Constituante de vouloir établir un « gouvernement représentatif absolu » où la Nation « délègue en masse » sa souveraineté (VII, 615). C'est selon lui ce qu'implique la rédaction de l'article et le fait que le projet du comité de constitution ne laisse à « la Nation aucun moyen constitutionnel d'exprimer une seule fois sa volonté sur ce que ses mandataires et ses délégués auront fait en son nom » (VII, 612). 
Robespierre s'étonne d'autre part que le roi soit désigné dans l'article 2 comme un « représentant », au même titre que le corps législatif alors qu'il est un « fonctionnaire public héréditaire » (VII, 612).

Le roi, précise-t-il, n'est « pas le représentant de la Nation », car « l'idée de représentant suppose nécessairement un choix du peuple [...]. Le hasard seul vous le donne et non votre choix. » (VII, 613). En conclusion de son intervention, Robespierre demande, pour éviter toute confusion, «qu'au mot pouvoir soit substitué celui fonction $»^{3}$ et que « le roi soit appelé le premier fonctionnaire public, le chef du pouvoir exécutif, mais point du tout le représentant de la Nation » (VII, 614).

Ce combat du « côte gauche » de l'Assemblée est alors récurrent. Le « côté droit » s'efforce en effet de transférer le pouvoir du peuple souverain à ses représentants, parmi lesquels elle compte donc le roi, une manière de reconstituer la puissance souveraine dont il a été dépossédé mais en la partageant avec l'Assemblée. C'est également la fonction du droit de veto qui est attribué à Louis XVI et contre lequel Robespierre a également bataillé. Dans le discours qu'il n'a pu prononcer (septembre 1789) et où il dénonce cette mesure, Robespierre définit la fonction du roi en ces termes : il s'agit de «l'homme [revêtu du pouvoir exécutif ] établi par la Nation, pour faire exécuter les volontés de la Nation » (VI, 87). Le fait que ce pouvoir exécutif soit établi par la Nation, signifie, précise Robespierre, que « tous ceux qui gouvernent, et par conséquent les rois eux-mêmes, ne sont que les mandataires et les délégués du peuple ; que les fonctions de tous les pouvoirs politiques, et par conséquent de la royauté, sont des devoirs publics, et non des droits personnels ni une propriété particulière » (VI-88). Quant à la «volonté de la Nation » que le roi doit exécuter, elle consiste dans la Déclaration des droits de l'homme et du citoyen.

Louis XVI utilise son titre de roi pour tenter de se placer au dessus de la Nation et d'échapper aux obligations imposées au pouvoir exécutif - borné par la Déclaration - que lui attribue le pouvoir constituant. Au contraire, avec le côté gauche de l'Assemblée Constituante, Robespierre participe du travail politique qui consiste à ramener le roi au niveau d'un pouvoir constitué, en d'autres termes à forcer le roi à être un mandataire du peuple souverain, plus précisément à être son commis, comme il le précise le 18 mai 1790 dans son discours Sur l'attribution au roi du droit de paix et de guerre : il « est inexact de dire [que le roi est un] représentant de la nation. Le roi est le commis et le délégué de la nation pour exécuter les volontés nationales » (IV-364)4. Le fait de désigner le roi comme un commis vaut à Robespierre un rappel à l'ordre à la suite duquel il s'explique :

«Par commis, je n'ai voulu entendre que l'emploi suprême, que la charge sublime d'exécuter la volonté générale ; j'ai dit qu'on ne représente la Nation que quand on est spécialement chargé par elle d'exprimer sa volonté. Toute autre puissance, quelque auguste qu'elle soit, n'a pas le caractère de Représentant du Peuple (VI, 364). »

Ces différentes prises de position sur le statut du roi permettent de distinguer des usages dans le vocabulaire politique que mobilise Robespierre et la nature de la relation fiduciaire

3 Souligné dans le texte.

4 Souligné dans le texte. 
de ceux qui sont chargés par le peuple de porter sa confiance et qui peuvent la perdre. Le roi est un fonctionnaire public, un délégué, un commis du peuple qui est en charge du pouvoir exécutif. Mais il est aussi qualifié de mandataire, c'est-à-dire qu'il dispose d'un mandat dont il doit rendre compte, qualité qu'il partage avec les représentants du peuple dont il n'est pas, puisqu'il n'est pas choisi par le peuple et n'est pas chargé par lui d'exprimer sa volonté. En creux, nous disposons d'une définition du représentant du peuple : celui qui est élu pour exprimer la volonté du peuple souverain. Pourtant, trois années plus tard, le 16 juin 1793, au cours d'un autre débat constitutionnel, Robespierre juge que « le mot de représentant » est abusif et inapproprié pour qualifier les membres de la législature puisque "la volonté souveraine ne se représente pas ». Il rappelle que la loi devient loi seulement lorsque le peuple souverain l'accepte et exprime lui-même sa volonté, non lorsque ses élus en votent le projet, ce qui est prévu dans la Constitution de $1793^{5}$ :

« [...] le mot de représentant ne peut être appliqué à aucun mandataire du peuple, parce que la volonté ne peut se représenter. Les membres de la législature sont les mandataires à qui le peuple a donné la première puissance ; mais dans le vrai sens on ne peut pas dire qu'ils le représentent. La législature fait des lois et des décrets ; les lois n'ont le caractère de lois que lorsque le peuple les a formellement acceptées. Jusqu'à ce moment, elles n'étaient que des projets ; alors elles sont l'expression de la volonté du peuple. Les décrets ne sont exécutés avant d'être soumis à la ratification du peuple, que parce qu'il est censé les approuver : il ne réclame pas, son silence est pris pour une approbation. Il est impossible qu'un gouvernement ait d'autre principe. Ce consentement est exprimé ou tacite ; mais, dans aucun cas, la volonté souveraine ne se représente, elle est présumée. Le mandataire ne peut être représentant, c'est un abus de mot, et déjà en France on commence à revenir de cette erreur (IX, 569) ${ }^{6}$. »

On peut avancer l'hypothèse selon laquelle, sous la Constituante, l'enjeu politique étant de séparer clairement le roi de la fonction législative, il s'agit de l'exclure de la représentation, non de nier également la validité du mot « représentant» pour qualifier l'Assemblée, au risque de la confondre avec le roi dans leur commune position de mandataire. On pourrait également en conclure qu'au début de la Révolution, Robespierre aurait été partisan du « système représentatif », dans lequel les représentants ont la charge d'exprimer la volonté souveraine, et qu'il aurait abandonné le terme représentant pour lui préférer celui de mandataire. Suivant nos découpages contemporains, nous pourrions dès lors penser que

5 Article 58. - Le projet est imprimé et envoyé à toutes les communes de la République, sous ce titre : loi proposée. Article 59. - Quarante jours après l'envoi de la loi proposée, si, dans la moitié des départements, plus un, le dixième des Assemblées primaires de chacun d'eux, régulièrement formées, n'a pas réclamé, le projet est accepté et devient loi.

6 Souligné dans le texte. Robespierre emprunte ici à Rousseau : «La Souveraineté ne peut être représentée, par la même raison qu'elle ne peut être aliénée ; elle consiste essentiellement dans la volonté générale, et la volonté ne se représente point : elle est la même, ou elle est autre ; il n’y a point de milieu. Les députés du peuple ne sont donc ni ne peuvent être ses représentants, ils ne sont que ses commissaires ; ils ne peuvent rien conclure définitivement. Toute loi que le Peuple en personne n'a pas ratifiée est nulle ; ce n'est point une loi. » JeanJacques Rousseau, Du Contrat social, III-15. Notons que Robespierre n'a pas la même conception de la volonté générale que Rousseau. 
Robespierre serait favorable à la « démocratie représentative », mais que cherchant l'appui du mouvement populaire en 1793, il mettrait en avant la « démocratie directe » à laquelle est associé le terme mandataire. La thèse, en son temps développée par l'historien Albert Soboul selon laquelle Robespierre rallierait le mouvement populaire pour des raisons stratégiques est en effet courante.

Or, en dépit de sa remarque de 1793, Robespierre emploie indifféremment les mots représentant et mandataire tout au long de la Révolution. Par exemple, le préambule de son projet de Déclaration des droits qu'il présente le 24 avril 1793, donc quelques semaines avant le débat de juin 1793, débute en ces termes : «Les Représentants du Peuple Français réunis en Convention nationale, reconnaissant que les lois humaines qui ne découlent point des lois éternelles de la justice et de la raison ne sont que des attentats de l'ignorance ou du despotisme contre l'humanité... ». En revanche il n'utilise que le terme mandataire dans les articles qui suivent, jamais celui de représentant .

On ne peut pas en déduire que Robespierre qualifierait de représentants les élus du peuple qui disposent du pouvoir constituant (« Les Représentants du Peuple Français réunis en Convention nationale ») et de mandataires ceux qui exercent leurs fonctions alors que le pouvoir est constitué (décrit dans le corps de la Déclaration) puisque, là encore, les membres de toutes les assemblées, qu'elles soient ou non constituantes, apparaissent indistinctement sous sa plume comme des mandataires ou des représentants. Un règle semble cependant devoir être retenue : qu'il s'agisse de représentants ou de mandataires, on ne peut pas déléguer la souveraineté, seulement une partie de l'exercice du pouvoir législatif, le peuple souverain gardant la main au-delà de l'élection. Ceux que Robespierre nomme communément les représentants du peuple sont de fait ramenés au statut de mandataires du peuple. Si le vocabulaire n'est pas fixé, les principes le sont. L'article 14 de son projet de Déclaration les synthétise : «Le peuple est souverain : le gouvernement est son ouvrage et sa propriété, les fonctionnaires publics sont ses commis. Le peuple peut, quand il lui plaît, changer son gouvernement et révoquer ses mandataires. »

\section{Le droit à l'existence politique : donner et retirer sa confiance}

La citoyenneté est la principale condition de la confiance du peuple vis-à-vis de ses mandataires si elle consiste dans la possibilité de contrôler et de reprendre, à tout moment, l'exercice des fonctions déléguées, suivant la terminologie de Robespierre. Cela est d'autant plus nécessaire que les « fonctionnaires publics [...] sont naturellement enclins à s'identifier eux-mêmes avec l'autorité publique qui leur est confiée ; ils se croient propriétaires de ce dépôt, et en disposent sans scrupule au profit de leur vanité, de leur ambition, et de leur cupidité ; ils mettent sans façon leurs personnes à la place de la nation » (IV-147). Or, avant la révolution du 10 août 1792, le statut de citoyen actif est réservé à une partie de la population. Robespierre dénonce donc la rupture de confiance liée au suffrage censitaire et plus largement une politique qui est menée en faveur des propriétaires. Cette rupture du contrat justifie selon lui l'insurrection du peuple souverain contre ses « mandataires infidèles ».

Le système censitaire mis en œuvre au cours des premières années de la Révolution repose sur la distinction entre les citoyens actifs et les citoyens passif théorisée par Sieyès et adoptée par l'Assemblée le 29 octobre 1789. Avec la « délégation de pouvoir à perpétuité », 
c'est l'une des principales modalités de la réduction de l'emprise du peuple souverain sur ses représentants.

Les citoyens passifs, explique Sieyès, «peuvent jouir des avantages de la société », mais ils n'ont «pas droit à prendre une part active dans la formation des pouvoirs publics » que seuls les citoyens actifs possèdent. Tous les habitants du pays jouissent donc des droits de citoyens passifs, «tous ont droit à la protection de leur personne, de leur propriété, de leur liberté », mais seuls ceux qui « contribuent à l'établissement public sont comme les vrais actionnaires de la grande entreprise sociale » : « eux seuls sont les véritables citoyens actifs, les véritables membres de l'association. » Les femmes, les enfants, les étrangers et ceux qui ne contribuent pas, par leur richesse, « ne doivent point influencer activement sur la chose publique »(Sieyès,1789, 36-37). Les droits politiques qui consistent à voter, être éligible aux fonctions publiques, participer à la garde nationale sont donc conditionnés par le fait de contribuer, au sens matériel du terme, à la richesse de la société.

Les nombreuses prises de parole de Robespierre contre la politique censitaire consistent systématiquement à rappeler le contrat aux mandataires du peuple, car celui-ci implique que l'exercice des droits politiques soit attribué à tous les citoyens : le droit «à la protection de leur personne, de leur propriété, de leur liberté »- pour reprendre les termes de Sieyès - est intimement lié au contrôle de l'effectivité de ce droit. Robespierre rappelle le contrat dès la première intervention qu'il consacre à cette question, le 22 octobre 1789, le jour où la distinction entre actifs et passifs est mise en débat à l'Assemblée :

«La constitution [en l'occurrence la Déclaration des droits dans son article 3] établit que la souveraineté réside dans le peuple, dans tous les individus du peuple. Chaque citoyen a donc droit de concourir à la loi par laquelle il est obligé, et à l'administration de la chose publique qui est la sienne. Sinon il n'est pas vrai que tous les hommes sont égaux en droits, que tout homme est citoyen (VI-131). »

Le rappel du contrat structure également son discours contre le décret dit du marc d'argent ${ }^{7}$ d'avril 1791 :

«Pourquoi sommes-nous rassemblés dans ce temple des lois ? Sans doute pour rendre à la Nation française l'exercice des droits imprescriptibles qui appartiennent à tous les hommes. Tel est l'objet de toute Constitution politique. Elle est juste, elle est libre, si elle le remplit ; elle n'est qu'un attentat contre l'humanité, si elle le contrarie. Vous avez vous-mêmes reconnu cette vérité d'une manière frappante, lorsqu'avant de com-

7 «Sur la nécessité de révoquer les décrets qui attachent l'exercice des droits du citoyen à la contribution du marc d'argent ou d'un nombre déterminé de journées d'ouvrier ». Suivant le premier projet de la Constituante, il fallait acquitter un impôt annuel d'une valeur d'un «marc d'argent », (soit 52 livres, une livre correspondant au salaire d'un moissonneur pour une journée de travail) pour être éligible à la fonction de député. Cette barrière de cens sera finalement abandonnée et déplacée vers les « électeurs », c'est-à-dire ceux qui sont élus par les citoyens actifs et qui vont à leur tour élire les représentants du peuple. Pour être électeur il faut posséder un bien évalué à 150 ou 200 journées de travail, selon les cas, ou en louer un qui a une valeur de 100 à 150 journées de travail, suivant la taille des villes, ou de 400 journées de travail à la campagne. Les gros propriétaires contrôlent donc l'élection. 
mencer votre grand ouvrage, vous avez décidé qu'il fallait déclarer solennellement ces droits sacrés, qui sont comme les bases éternelles sur lesquelles il doit reposer ; «Tous les hommes naissent et demeurent libres et égaux en droits. La souveraineté réside essentiellement dans la Nation. La loi est l'expression de la volonté générale. Tous les citoyens ont le droit de concourir à sa formation, soit par eux-mêmes, soit par leurs représentants, librement élus. Tous les citoyens sont admissibles à tous les emplois publics, sans aucune autre distinction que celle de leur vertu et de leurs talents. »Voilà les principes que vous avez consacrés ; il sera facile maintenant d'apprécier les dispositions que je me propose de combattre, il suffira de les rapprocher de ces règles invariables de la société humaine (VII, 161). »

Après avoir comparé les principes de la Déclaration et le dispositif censitaire de la Constitution, Robespierre conclut à trois reprises que toutes ces dispositions fondées sur la distinction entre passif et actif « sont donc essentiellement anticonstitutionnelles et antisociales » (VII, 161) parce qu'elles contredisent les principes déclarés en dépossédant le peuple souverain :

«La Nation est-elle souveraine quand le plus grand nombre des individus qui la composent est dépouillé des droits politiques qui constituent la souveraineté ? Non, et cependant vous venez de voir que ces mêmes décrets les ravissent à la plus grande partie des Français. Que serait donc votre Déclaration des droits si ces décrets pouvaient subsister? Une vaine formule. Que serait la Nation? Esclave : car la liberté consiste à obéir aux lois qu'on s'est données, et la servitude à être contraint de se soumettre à une volonté étrangère. Que serait votre Constitution ? Une véritable aristocratie. Car l'aristocratie est l'état où une partie des citoyens est souveraine et le reste est sujet, et quelle aristocratie ! La plus insupportable de toutes, celle des riches (VII, 162). »

Dans une autre de ses interventions contre le marc d'argent ${ }^{8}$, il souligne par ailleurs l'illégalité de l'usage des pouvoirs confiés lorsqu'ils permettent au fidéicommis de déposséder ses commettants :

«Je demande maintenant si vous, qui êtes arrivés ici sans titre, et qui tenez vos pouvoirs de ces hommes-là, dont une grande partie n'atteignait pas la condition que vous leur imposez; je vous demande si vous pouvez vous servir des pouvoirs qu'ils vous ont confiés, et si vous pouvez leur dire : le jour où vous nous avez investis du pouvoir de défendre et de garder vos lois, ce jour-là, vous l'avez perdu : vous ne rentrerez plus dans ces assemblées où vous nous avez donné votre confiance (VII 622). »

Les citoyens actifs ne représentent environ que $70 \%$ des hommes de plus de 25 ans. L'accès au suffrage est donc nettement plus restreint que pour l'élection des députés aux

8 Sur le marc d'argent et sur le cens électoral, 11 août 1791. 
États généraux où chaque «feu » (chaque foyer) était représenté, une élection dont les membres de la Constituante tirent leur légitimité.

Toute entrave à l'exercice de la citoyenneté est de fait une entorse au contrat par lequel sont déclarés les droits de l'homme et du citoyen. « Vous ne pouvez pas, rappelle Robespierre au constituants, porter atteinte vous-même à la garantie de la liberté, de la justice, de l'égalité exacte que vous avez promise par la constitution ; parce que vous ne pouvez pas, de la manière la plus formelle et la plus évidente, effacer ces principes fondamentaux de la déclaration des droits des hommes et des citoyens, que vous avez reconnus comme la base de votre constitution » (VII, 621).

Si tel est le cas, la société n'est pas constituée puisque « la garantie des droits » n'est pas assurée, comme le stipule l'article 16 de la Déclaration de $1789^{9}$. Chaque contractant peut dès lors exercer son droit de résistance à l'oppression. Le désordre, explique Robespierre, ne résulte pas de l'insurrection d'un peuple qui recouvre ses droits, mais de l'action d'un gouvernement tyrannique qui attente aux principes qu'il est censé garantir.

La politique économique libérale mise en œuvre depuis 1789 favorise les propriétaires et la spéculation. Elle porte atteinte au droit à l'existence et constitue également une rupture du contrat. Robespierre soutient donc les insurrections populaires qui visent à faire baisser le prix des subsistances - la taxation - par lesquelles le peuple censure un pouvoir législatif qui génère le désordre :

«Les fonctionnaires publics de tous les pays commettent assez généralement, à cet égard, une erreur aussi funeste que commune. Ils ont coutume de rejeter sur la perversité des peuples les désordres de la société ; ils les accusent de rébellion, lorsqu'eux seuls sont coupables d'orgueil et d'injustice, et de tous temps ce grand procès fut décidé contre les peuples ; car ce sont les fonctionnaires publics qui le jugent. [...] Ceux qu'ils oppriment osent-ils se plaindre ? Ils crient à la désobéissance, à la rébellion. Ils invoquent le respect dû aux autorités constituées ; ils jurent que la tranquillité publique est troublée ; il les immolent au nom de la loi. [...] La tranquillité, c'est l'ordre public, c'est l'harmonie sociale. Peut-elle exister sans la justice, sans la liberté, sans le bonheur? Quels sont ceux qui la troublent? Sont-ce les tyrans qui violent les droits des peuples, ou les peuples qui les réclament? Peuples, tyrans, voilà toute votre cause ; que la raison, que l'humanité la juge une fois, et non la force et le despotisme (IV, 147-149) ${ }^{10}$. »

Le 2 décembre 1792, dénonçant les Girondins qui poursuivent la même politique économique favorable à la liberté du commerce des grains, Robespierre rappelle de nouveau qu'un peuple ne se révolte jamais contre les lois qu'il aime :

« [...] le peuple est naturellement droit et paisible ; il est toujours guidé par une intention pure; les malveillants ne peuvent le remuer, s'ils ne lui présentent un motif

9 «Toute Société dans laquelle la garantie des Droits n'est pas assurée, ni la séparation des Pouvoirs déterminée, n'a point de Constitution ».

10 «Sur le respect dû aux lois et aux autorités constituées », Le Défenseur de la Constitution, n5, juin 1792. 
puissant et légitime à ses yeux. Ils profitent de son mécontentement plus qu'ils ne le font naître ; et quand ils le portent à des démarches inconsidérées, par le prétexte des subsistances, ce n'est que parce qu'il est disposé à recevoir ces impressions, par l'oppression et par la misère. Jamais un peuple heureux ne fut un peuple turbulent. Quiconque connaît les hommes, quiconque connaît surtout le peuple français, sait qu'il n'est pas au pouvoir d'un insensé ou d'un mauvais citoyen, de le soulever sans aucune raison, contre les lois qu'il aime, encore moins contre les mandataires qu'il a choisis, et contre la liberté qu'il a conquise. C'est à ses représentants à lui témoigner la confiance qu'il leur donne lui-même, et de déconcerter la malveillance aristocratique, en soulageant ses besoins, et en calmant ses alarmes (IX, 116). »

La révolution du 10 août 1792 qui renverse Louis XVI et la Constitution censitaire, comme celle des 31 mai-2 juin 1793 qui met fin à la Convention girondine, retirent la confiance donnée à des mandataires infidèles.

\section{Le contrôle politique des biens qui permettent d'exister}

Dans son discours Sur les principes de morale politique qui doivent guider la Convention nationale dans l'administration intérieure de la République du 17 pluviôse an II (5 février 1794), Robespierre définit la démocratie en ces termes :

« La démocratie n'est pas un état où le peuple, continuellement assemblé, règle par lui-même toutes les affaires publiques, encore moins celui où cent mille fractions du peuple, par des mesures isolées, précipitées et contradictoires, décideraient du sort de la société entière : un tel gouvernement n'a jamais existé, et il ne pourrait exister que pour ramener le peuple au despotisme.

La démocratie est un état où le peuple souverain, guidé par des lois qui sont son ouvrage, fait par lui-même tout ce qu'il peut bien faire, et par des délégués tout ce qu'il ne peut faire lui-même $(\mathrm{X}, 353)$. »

Si le peuple souverain possède le pouvoir législatif, il ne légifère pas directement mais à travers les représentants qu'il s'est donnés. Dans une démocratie - c'est-à-dire dans une république, ces deux mots étant synonymes dit Robespierre - le peuple fait donc par ses représentants ce pour quoi il est souverain, ce qui ne va pas sans risque. En juin 1792 dans un article du Défenseur de la Constitution qu'il consacre au "respect dû aux lois et aux autorités constituées », Robespierre explique que «c'est seulement par fiction que la loi est l'expression de la volonté générale » lorsque l'exercice du pouvoir législatif est délégué : «Le législateur n'est point infaillible, fût-il le peuple lui-même. Les chances de l'erreur sont bien plus nombreuses encore, lorsque le peuple délègue l'exercice du pouvoir législatif à un petit nombre d'individus ; c'est-à-dire, lorsque c'est seulement par fiction que la loi est l'expression de la volonté générale (IV-145). »

Puisque le peuple exerce sa souveraineté à travers ses représentants lorsqu'il ne peut pas l'exercer lui-même, ceux-ci doivent donc représenter au mieux la volonté du peuple. C'est en cela que les représentants du peuple doivent être impérativement vertueux. Cette vertu 
politique - la vertu républicaine au sens de Montesquieu ${ }^{11}$ - consiste, précise Robespierre, dans « l'amour de la patrie et de ses lois », en d'autres termes dans l'amour de l'égalité : « l'essence de la république ou de la démocratie est l'égalité, il s'ensuit que l'amour de la patrie embrasse nécessairement l'amour de l'égalité (X, 353). »

Pour Robespierre, la richesse et les «passions ruineuses » qui l'accompagnent, mais aussi le fait d'être propriétaire et donc indépendant, n'est pas au service de la vertu politique, c'est-à-dire une vertu dans laquelle la confiance peut-être politiquement fondée, contrairement à la frugalité. Il le rappelle dans ses interventions contre le suffrage censitaire :

«Le comité dit : il faut une garantie de l'indépendance et de la pureté des intentions de ceux qui devront choisir les représentants de la Nation. D’abord, messieurs, je conviens qu'il faut une garantie ; mais cette garantie est-ce la contribution, est-ce la fortune qui la donnent ? Est-il vrai que la probité, que les talents se mesurent réellement sur la fortune ? Je dis que l'indépendance, la véritable indépendance, est relative, non pas à la fortune, mais aux besoins, mais aux passions des hommes ; et je dis qu'un artisan, qu'un laboureur qui paie les dix journées de travail exigées par vos précédents décrets, est plus indépendant qu'un homme riche, parce que ses désirs et ses besoins sont encore plus bornés que sa fortune, parce qu'il n'est point accablé de toutes ces passions ruineuses, enfants de l'opulence (VII, 620-621). »

Le fait d'être propriétaire est d'autant moins une qualité que la garantie du droit à l'existence implique un contrôle politique de l'usage des propriétés qu'il est vain d'attendre des propriétaires.

La pensée républicaine de Robespierre, ce qu'il nomme une économie politique populaire (Gauthier, 1988 et 1992), repose sur une conception de la république dont la fonction est de garantir le droit naturel à l'existence et à la liberté (article 2 de son projet de Déclaration), ces deux droits étant intimement liés - il n'y a pas de liberté possible si le droit à l'existence de chacun n'est pas garanti - , et c'est au peuple souverain qu'il confie le soin de contrôler si cette garantie est effective, c'est-à-dire si ses mandataires font de «bonnes lois ».

Dans son discours du 2 décembre 1792 sur les subsistances, au cours de l'un des débats sur la liberté du commerce de grains, Robespierre énonce les grands principes qu'il reformule quelques mois plus tard dans sa Déclaration des droits et qui sont l'objet d'une politique républicaine :

«Quel est le premier objet de la société ? C'est de maintenir les droits imprescriptibles de l'homme. Quel est le premier de ces droits ? Celui d'exister. La première loi sociale est donc celle qui garantit à tous les membres de la société les moyens d'exister ; toutes les autres sont subordonnées à celle-là ; la propriété n'a été instituée ou garantie que pour la cimenter ; c'est pour vivre d'abord que l'on a des propriétés.

11 «Pour l'intelligence des quatre premiers livres de cet ouvrage, il faut observer que ce que j'appelle la vertu dans la république est l'amour de la patrie, c'est-à-dire l'amour de l'égalité. Ce n'est point une vertu morale, ni une vertu chrétienne, c'est la vertu politique; et celle-ci est le ressort qui fait mouvoir le gouvernement républicain, comme l'honneur est le ressort qui fait mouvoir la monarchie. J'ai donc appelé vertu politique l'amour de la patrie et de l'égalité ». Montesquieu, De l'esprit des lois, « Avertissement au lecteur ». 
Il n'est pas vrai que la propriété puisse jamais être en opposition avec la subsistance des hommes (IX-112). »

Robespierre ne limite pas la notion de propriété aux biens matériels. Elle consiste dans ce qu'il nomme l'idée générale de propriété qui concerne aussi les droits attachés à la personne c'est-à-dire, écrit Robespierre, «ma liberté, ma vie, le droit d'obtenir sûreté ou vengeance pour moi et pour ceux qui me sont chers, le droit de repousser l'oppression, celui d'exercer librement toutes les facultés de mon esprit et de mon cœur » (VII,164-165). Dès le début de la Révolution, avec le côté gauche, Robespierre mobilise cette idée générale de propriété contre la politique censitaire qui vise à faire de la propriété matérielle la condition de l'exercice des droits politiques :

«Par un étrange abus des mots, [les riches, les hommes puissants] ont restreint à certains objets l'idée générale de propriété ; ils se sont appelés seuls propriétaires ; ils ont prétendu que les propriétaires seuls étaient dignes du nom de citoyen ; ils ont nommé leur intérêt particulier l'intérêt général, et pour assurer le succès de cette prétention, ils se sont emparés de toute la puissance sociale (VII, 165). »

Robespierre distingue les biens qui permettent de vivre et ceux qui n'ont pas pour fonction de garantir le droit à l'existence : « les grossiers habits qui me couvrent, l'humble réduit où j'achète le droit de me retirer et de vivre en paix, le modique salaire avec lequel je nourris ma femme, mes enfants, tout cela, je l'avoue, ne sont point des terres, des châteaux, des équipages » (VII, 164). A la différence de celle des biens qui permettent de vivre, la propriété « des terres, des châteaux, des équipages », n'est pas pour Robespierre un droit naturel inaliénable, attaché à la personne, mais une convention sociale, un droit attribué par les sociétés et qui doit donc être régulé par les sociétés. Cette régulation se fait en fonction du principe qui fonde la république : le respect du droit naturel à l'existence.

La liberté du propriétaire est ainsi limitée par la liberté d'autrui. Le 24 avril 1793, Robespierre dénonce en ces termes le projet de Déclaration des droits de Condorcet favorable à la liberté illimitée du propriétaire et qui ignore le droit à l'existence :

«En définissant la liberté le premier des biens de l'homme, le plus sacré des droits qu'il tient de la nature, vous avez dit avec raison qu'elle avait pour borne les droits d'autrui : pourquoi n'avez-vous pas appliqué ce principe à la propriété, qui est une institution sociale ? Comme si les lois éternelles de la nature étaient moins inviolables que les conventions des hommes. Vous avez multiplié les articles pour assurer la plus grande liberté à l'exercice de la propriété, et vous n'avez pas dit un seul mot pour en déterminer le caractère légitime ; de sorte que votre Déclaration paraît faite, non pour les hommes, mais pour les riches, pour les accapareurs, pour les agioteurs et pour les tyrans (IX-461).»

Robespierre met en avant une conception fiduciaire de la propriété, c'est-à-dire conditionnelle et limitée. Elle relève de la politique et ne peut être laissée au libre jeu des intérêts privés, elle est « encastrée » (embedded) au sens de Polanyi. La république se réserve le 
droit de statuer sur la propriété en fonction du principe qui fonde cette république : le droit à l'existence comme condition nécessaire de la liberté.

Le programme égalitaire dont Robespierre est l'un des porte-parole et selon lequel chacun doit avoir un droit égal à la liberté, ne condamne pas la propriété individuelle si elle est limitée par le droit à l'existence d'autrui, c'est-à-dire si elle se conforme à la conception républicaine de la liberté fondée sur la non-domination.

Les subsistances, et plus largement tout ce qui permet de garantir l'existence, sont considérées comme une propriété commune qui ne peut pas être abandonnée au marché. Robespierre le rappelle dans son discours du 2 décembre 1792 :

«Les aliments nécessaires à l'homme sont aussi sacrés que la vie elle-même. Tout ce qui est indispensable pour la conserver est une propriété commune à la société entière. Il n'y a que l'excédent qui soit une propriété individuelle, et qui soit abandonné à l'industrie des commerçants. Toute spéculation mercantile que je fais aux dépens de la vie de mon semblable n'est point un trafic, c'est un brigandage et un fratricide (IX, 112-113). »

Les subsistances sont une propriété commune, au sens de la res publica romaine, une propriété qui est commune parce qu'elle a été déclarée telle, par un acte politique, ce qui la distingue de la res communes qui est un bien commun, appartenant à tous en raison de sa nature, et non par choix politique, comme l'eau ou l'air. L'administration de cette propriété commune n'est pas l'affaire d'une bureaucratie centrale mais celle des citoyens. Elle est organisée par la loi du 14 frimaire an II (4 décembre 1793) qui institue le «Gouvernement révolutionnaire ». Dans ce cadre, elle attribue aux municipalités, l'échelon administratif le plus proche des habitants, et aux comités révolutionnaires (ou de surveillance) qui sont élus localement, le pouvoir exécutif des lois révolutionnaires (section II, article 8), en particulier celle dite du maximum. Celle-ci ne concerne pas seulement l'encadrement du prix des produits alimentaires mais aussi celui des matières premières dont dépendent les artisans pour leur existence et les salaires qu'il faut également surveiller afin de maîtriser l'inflation.

Il ne s'agit donc pas d'une économie contrôlée par le haut comme on le pense fréquemment, mais d'une économie sous contrôle populaire. Il ne s'agit pas non plus de mesures de circonstance, liées à la guerre intérieure et extérieure. Il s'agit plutôt, en dépit des circonstances, de maintenir le cap de la liberté républicaine fondée sur l'action du peuple souverain. Ces mesures sont cohérentes avec le principe selon lequel il est nécessaire, dans une république, de laisser le pouvoir exécutif au plus près des citoyens, comme Robespierre l'évoque le 10 mai 1793 :

«Fuyez la manie ancienne des gouvernements de vouloir trop gouverner ; laissez aux individus, laissez aux familles le droit de faire ce qui ne nuit point à autrui ; laissez aux communes le pouvoir de régler elles-mêmes leurs propres affaires en tout ce qui ne tient pas essentiellement à l'administration générale de la République ; en un mot, rendez à la liberté individuelle tout ce qui n'appartient pas naturellement à l'autorité publique, et vous aurez laissé d'autant moins de prise à l'ambition et à l'arbitraire (IX-501). » 
Saint-Just le rappelle cinq jours plus tard en indiquant que « la souveraineté de la nation réside dans les communes » (Saint-Just, 2004, 582). Appliqué à l'économie, ce principe permet d'établir une « loi populaire », que Saint-Just appelle de ses vœux le 29 novembre 1792, « qui mette la liberté du commerce sous la sauvegarde du peuple-même, selon le génie de la république » (Saint-Just, 2004, 498). Pour sa part, Coupé de l'Oise, également proche de Robespierre, juge qu' « il faut individualiser les opérations de commerce et les laisser toujours sous les yeux des citoyens » (Coupé, 1793, 164). Au cours du débat du 21 août 1793 à la Convention, cette position est réaffirmée par Duhem au côté de Coupé :

« Je m'oppose à ce qu'on mette en administration ou en régie les subsistances du peuple ; ce sont toutes ces administrations qui nous font mourir de faim. C'est là que se nichent tous les intrigants, les voleurs de toute espèce, et les dilapidateurs les plus effrontés et les plus coupables. (Applaudissements.) [...] Vous ne pouvez placer toute votre confiance que dans la masse populaire ; c'est là seulement qu'on trouve la véritable probité. C'est donc au peuple lui-même qu'il faut laisser le soin d'assurer les subsistances ${ }^{12}$.»

Le peuple ne faisait rien d'autre par sa pratique de la taxation. La loi du 14 frimaire an II - comme de nombreuses lois votées sous la Convention montagnarde (Gross, 2016) encadre des pratiques, elle ne les crée pas.

Le républicanisme que Robespierre désigne sous la notion d' "économie politique populaire » repose sur une conception fiduciaire de l'exercice du pouvoir, mais également sur une conception fiduciaire de la propriété. Cette " économie politique populaire », explique Robespierre, consiste à placer «dans la vertu du peuple et dans l'autorité du souverain le contre-poids nécessaire des passions du magistrat et de la tendance du gouvernement à la tyrannie » (IX-507), une tyrannie qui se caractérise par l'atteinte aux droits naturels de l'homme dont le premier est le droit à l'existence. Celui-ci n'est pas seulement matériel mais aussi et surtout politique, puisque c'est le droit à l'existence politique qui constitue la principale garantie du droit à l'existence matérielle. Il permet de contrôler les usages des biens qui sont indispensables à la vie. Ces biens sont «une propriété commune à la société entière », au même titre que le gouvernement (au sens large) est la propriété du peuple souverain. Ce modèle républicain, fondé sur le contrôle populaire de l'exercice du pouvoir et de l'usage de la propriété - et la répression des prévaricateurs et des accapareurs qu'il implique, surtout en période de guerre intérieure et extérieure -, est dénoncé par la Convention thermidorienne qui le qualifie « d'organisation de l'anarchie » et de «système de la terreur ». Avec la Constitution directoriale votée en 1795, elle lui substitue une république aristocratique établie sur le suffrage censitaire et le pouvoir des propriétaires. Le contrat est refondu de telle sorte que des « démagogues »comme Robespierre est alors désigné - ne puissent plus le brandir et appeler le peuple à l'insurrection. La Déclaration des droits de l'homme et du citoyen est alors vidée de son contenu (Gauthier, 1992 ; Bosc, 2016).

12 Archives parlementaires, t 72, p. 748-749. 


\section{Références}

Bosc Y. (2016), La terreur des droits de l'homme. Le républicanisme de Thomas Paine et le moment thermidorien, Paris : Kimé.

Coupé J.-M. (1793), Principes du décret à porter sur le rétablissement de l'ordre dans les subsistances, cité par Guy Ikni, « Jean-Michel Coupé, curé jacobin », La guerre du blé au XVIIIe siècle. La critique populaire contre le libéralisme économique, Florence Gauthier et Guy Ikni (dir.), Montreuil : Les Éditions de la Passion, 1988.

Domènech A. (2009), « Droit, droit naturel et tradition républicaine moderne », Républicanismes et droit naturel. Des humanistes aux révolutions des droits de l'homme et du citoyen, Marc Belissa, Yannick Bosc et Florence Gauthier (dir.), Paris : Kimé, 2009.

Gauthier F. (1988), « De Mably à Robespierre. De la critique de l'économique à la critique du politique », La guerre du blé au XVIIIe siècle. La critique populaire contre le libéralisme économique, Florence Gauthier et Guy Ikni (dir.) Montreuil : Les Éditions de la Passion.

Gauthier F. (1992), Triomphe et mort du droit naturel en Révolution. 1789-1795-1802, Paris : PUF, (rééd. Syllepses, 2014).

Guilhaumou J. (1989), La langue politique et la Révolution française. De l'événement à la raison linguistique, Paris : Méridiens Klincksieck.

Gross J.-P. (2016), Égalitarisme jacobin et droits de l'homme, (2000), rééd., Paris : Kimé.

Locke J. (1690), Deuxième traité du gouvernement civil, trad. Mazel (1795).

Robespierre M. (1910-2007), Euvres de Maximilien Robespierre, 11 vol., Paris, SER.

Saint-Just L.-A. (2004), Euvres complètes, Paris. Gallimard, 2004, p. 582.

Sieyès E.-J. (1789), Reconnaissance et exposition raisonnée des droits de l'homme et du citoyen, Paris : Baudouin. 
